\title{
De-escalation: a survey of clinical staff in a secure mental health inpatient service
}

Nutmeg Hallett and Geoffrey L. Dickens

This is the peer reviewed version of the following article:

Hallett, N. and Dickens, G.L. 2015. De-escalation: a survey of clinical staff in a secure mental health inpatient service. International Journal of Mental Health Nursing. 24(4): pp.324-333. doi: 10.1111/inm.12136

which has been published in final form at http://dx.doi.org/10.1111/ inm.12136. This article may be used for non-commercial purposes in accordance with Wiley Terms and Conditions for Self-Archiving. 


\section{De-escalation: A survey of clinical staff in a secure mental health inpatient service}

Nutmeg Hallett, RMN, BNurs(Hons)

Geoffrey L. Dickens RMN BSc(Hons) PGDipN MA PhD

Study carried out at: St Andrew's, Billing Road, Northampton, NN1 5DG, UK

Corresponding author: Geoffrey L. Dickens. Professor of Mental Health Nursing, Abertay University, Kydd Building, Bell Street, Dundee. DD1 1HG, email:

g.dickens@abertay.ac.uk 


\begin{abstract}
De-escalation is an important tool for preventing aggression in inpatient settings but definitions vary, and there is no clear practice guideline. We aimed to identify how clinical staff define and conceptualise de-escalation; which de-escalation interventions they would use in aggressive scenarios; and their beliefs about the efficacy of de-escalation interventions. A questionnaire survey $(N=72)$ was conducted using open and closed questions; additionally, clinical vignettes describing conflict events were presented for participants to provide their likely clinical response. Qualitative data were subject to thematic analysis. The major themes that de-escalation encompassed were: communication, tactics, de-escalator qualities, assessment and risk, getting help, and containment measures. Different types of aggression were met with different interventions. Half of participants identified PRN medication as a deescalation intervention, and $15 \%$ stated that seclusion, restraint and emergency intramuscular medication could be de-escalation interventions. Those interventions seen as most effective were the most commonly used. Clinical staff's views about de-escalation, and their deescalation practice, may differ from optimal practice. Use of containment measures and PRN medication where de-escalation is more appropriate could have a negative impact; work is needed to promote understanding and use of appropriate de-escalation interventions based on a clear guideline.
\end{abstract}

\title{
Key words:
}

Aggression, prevention and control, forensic nursing, mental health, inpatients 


\section{Introduction}

In the UK, clinical staff have a legal duty to respond to patient violence and aggression proportionately to the threat posed (Paterson et al., 2004). National clinical guidelines state that coercive interventions including seclusion, restraint and rapid tranquilisation should only be considered once de-escalation strategies have failed (National Institute for Clinical Excellence, 2005). De-escalation has been described as: 'a complex range of skills designed to abort the assault cycle during the escalation phase, and these include both verbal and non-verbal communication skills' (National Institute for Clinical Excellence, 2005:8). De-escalation involves the use of 'verbal and physical expressions of empathy, alliance and nonconfrontational limit setting that is based on respect' (Cowin, 2003: p. 65). However, there is currently no widely accepted gold standard definition of de-escalation nor clear guidance on the practices that clinical staff should use (Roberton et al., 2012). Various authorities have identified and described elements of de-escalation, many providing a list of the basic 'rules' (e.g., Stevenson, 1991; National Institute for Clinical Excellence [NICE], 2005; Richter, 2006). The NICE guidelines identify the need for identification of triggers, and for staff to be aware of their own verbal and non-verbal behaviour, and repeatedly refer to 'de-escalation techniques' with little clarification of what these techniques are.

A number of theoretical models of de-escalation have been proposed which can be categorised under two headings. The first type view de-escalation as an unfolding process occurring over distinct phases. Leadbetter and Paterson's (1995) model is based on the assault cycle which traces the development of an aggressive incident from the aggressor's baseline behaviour through five sequential phases: trigger, escalation, crisis, recovery and post-crisis depression. For each phase, the authors describe the dominant emotion of the aggressor, make recommendations about the aim and focus of staff intervention, and the skills and tactics to employ. Similarly, McDonnell (2010) has proposed a low arousal model of de-escalation based on four principles: decreasing staff demands on patients, avoidance of potentially arousing 
triggers (e.g. direct eye contact), avoidance of potentially arousing non-verbal behaviour (e.g. an aggressive stance), and challenging clinician beliefs about the management of aggression. This model comprises three phases: pre-crisis intervention, management of the critical incident, and post-incident recovery. Intervention strategies at each phase are recommended. A second type of de-escalation model focuses solely on the escalation phase of a potentially aggressive incident. Dix and Page's (2008) Assessment, Communication and Tactics (ACT) suggests a cyclical rather than linear approach comprising three interdependent components. Assessment involves consideration of the situational variables and the subject's responses; communication involves capturing the subject's attention, thus allowing continued access to the presenting problem; finally, specific tactics to reduce the likelihood of escalation include problem-solving, distraction, moving the patient, and removal of triggers. Each element of the model is to be continuously revisited by the de-escalator during the situation. Finally, as part of the Safewards (Bowers, 2014b) initiative to reduce conflict and containment in psychiatric settings, a model for de-escalation was designed to guide a range of interventions (Bowers, 2014a). The deescalation model focuses on communication strategies, but also identifies specific de-escalation tactics, and the desirable qualities and interpersonal skills of the de-escalator. The model identifies the de-escalation process as linear, moving through stages of de-limiting, and clarifying, to conflict resolution. De-escalator qualities, including emotional regulation and interpersonal skills such as empathy and respect, are suggested to influence the process throughout. A cluster randomised controlled trial of a range of Safewards interventions, including use of the de-escalation model, has demonstrated a statistically significant reduction in conflict and containment (Bowers, 2013); however it is not possible to separate out the specific effects of the de-escalation element of the intervention.

In addition to the theoretical models outlined above, a number of qualitative studies have explored de-escalation using observation, interviews and focus groups. Findings from these have been synthesised by Price and Baker (2012) who found seven themes: three relating 
to staff skills (characteristics of effective de-escalators, maintaining personal control, and communication skills), and four relating to the process of intervention (engaging with the patient, the timing of the intervention, safe conditions, and de-escalation strategies). Only four of the 11 studies reviewed obtained the views of unqualified ward staff even though they are often the group with most direct contact with patients. Interestingly, none of the studies reviewed appeared to define de-escalation during the research process, nor sought to obtain participants' definitions of the term. Furthermore, although some of these studies investigated effective de-escalation interventions, none appeared to identify which interventions are most frequently used.

\section{Rationale and aims}

The lack of consensus regarding a working definition of de-escalation hinders the provision of best practice guidelines for de-escalation, and is a potential barrier to effective research and education. The degree to which there is consensus among frontline clinical staff about what constitutes de-escalation, and which practices they would describe as de-escalation interventions, has potentially important implications. A theory-practice gap could have implications for interpretation of nursing documentation and the consistency and quality of care provided to patients, and would have important implications for training and education. The aim of this study was to explore the views of a range clinical staff about de-escalation. The specific objectives were to:

- identify how clinical staff define de-escalation

- describe the practices that staff identify as de-escalation interventions

- describe the interventions staff report using during low level conflict events

- identify which interventions staff believe constitute de-escalation

- examine which de-escalation interventions staff believe are most effective

\section{Materials and methods}

\section{Design}


A cross-sectional mixed-methods questionnaire survey design incorporating quantitative and qualitative elements.

\section{Setting and participants}

The study was conducted at St Andrew's, a large charitable provider of specialist secure inpatient mental health care. Data collection took place between July 2013 and February 2014. The aim of the study was exploratory and not to accurately estimate the proportions of staff holding different views, for which random sampling would have been more appropriate, we used purposive and convenience sampling. We wanted to capture the views of those most likely to have substantial contact time with patients in the ward setting. While we did not make any specific exclusion criteria in terms of occupation/profession our strategy was to recruit from among clinical staff on the ward or on ward-related training; specifically, $\mathrm{NH}$ visited a number of wards to recruit participants, and also approached staff who were attending annual mandatory training on Prevention and Management of Aggression and Violence (PMAV).

\section{Procedure}

The study was approved by the University of Northampton Research Ethics Committee and by hospital management. Participants were provided with a study questionnaire and a brief verbal explanation of the purpose of the study. Consent was implied from return of the completed questionnaire.

\section{Measures}

We developed a purpose-made 10-item instrument for this study, containing both open and closed questions in order to provide both qualitative and quantitative data. The questionnaire covered three areas. Section one explored participants' definitions of deescalation using an open-ended question ('What is de-escalation?'). Section two aimed to gather a corpus of data on views about de-escalation using a range of stimuli. This section included open-ended questions about the last time de-escalation was used and witnessed by the participant. Additionally, scenarios in the form of case vignettes were presented to participants 
who were asked 'What actions would you take?' Vignettes were developed about a range scenarios involving a range of aggressive behaviour commonly found in measures that have been developed to aid quantification of the type and severity of aggressive behaviour (e.g., Overt Aggression Scale, Yudofsky et al., 1986), namely: verbal aggression, self-harm/selfdirected aggression, physical aggression towards other people, physical aggression towards objects. In order to develop scenarios that required some form of intervention, but not necessarily more coercive measures, vignettes were calibrated against OAS criteria for second level severity (of the four levels available). The vignettes were discussed by the researchers, and reviewed by clinical and research colleagues until agreement was reached that all reflected level 2 scenarios. The vignettes are:

- Verbal aggression (VA): You hear shouting coming from the day area, so you go to see what is happening. When you get there you see Tom, one of the patients, waving his arms around and shouting that he wants to change the channel to watch his favourite TV programme. Tom seems to be directing his comments towards one of the other patients who is watching TV, saying to him 'You stupid idiot'.

- Self-harm (SH): You are checking Sadie, a patient who is on intermittent enhanced support (15 minutes). You find Sadie in her bedroom, head-butting the wall. She does not appear to be doing this hard enough to cause herself injury.

- Aggression towards people (AP): You are in the courtyard with a group of patients. You see one patient, Billy, grab the hair of another patient. The other patient cries out in pain but Billy does not let go.

- Aggression towards objects (AO): You are in the office when Jane, a patient, knocks on the door. You are on the phone, but you go to the door and tell Jane that you will attend to her as soon as you are finished on the phone. Jane slams the office door and then kicks a chair knocking it over. 
Section 3 of the questionnaire used closed-questions about a range of interventions in order to clarify participants' understanding of de-escalation e.g., 'which of the following is a deescalation intervention?' Participants were asked to complete the questionnaire sequentially so that information presented in closed questions in section 3 did not influence open-ended responses to sections 1 and 2 .

\section{Data analysis}

Each section of the survey was analysed separately. Responses to items in sections one and two requiring free text answers were subject to thematic analysis using Braun and Clarke's (2006) method. Data from section one and section two were transcribed into separate tables in Microsoft Excel. For each, an initial set of codes was developed and allocated to words and phrases in the data set. Data was then grouped by code and arranged into sets of higher level themes. Codes and themes were discussed and agreed throughout the analysis by GD and NH. We subsequently performed a frequency analysis of codes falling under each of the high level themes in terms of the presence or absence of the theme in each participant's response to each question. Data from responses in section three were entered into SPSS 18 and subject to descriptive statistics only.

\section{Results}

In total, 80 questionnaires were distributed and 72 were returned (response rate $90 \%$ ). Demographic details of the participants are presented in Table 1.

Table 1 about here

\section{Definitions of de-escalation}

Thematic analysis resulted in identification of three high level themes: objectives encompassed the aim or intention of de-escalation which was described as 'calming' or 'bringing down' the patient or situation, or 'preventing further escalation'; interventions described the specific the methods to be employed to achieve objectives and included verbal communication, relocation and distraction; finally, characteristics, were the idiosyncratic 
features of the individual and/or situation to be de-escalated, for example an angry patient or an over-stimulating environment.

\section{Views about de-escalation}

Thematic analysis of the free-text responses given to questions in section two resulted in six high level themes: communication, tactics, interpersonal skills, assessment / risk, getting help and containment measures.

Communication. This theme encompassed the verbal strategies that might be used to defuse the situation, and the channels through which this might be achieved. An attempt to open the channels of communication was described in almost half of all responses, most commonly talking to the patient or offering 'one to one' time.

Communication strategies included problem-solving ('They enquired about the nature of the problem and what could be done to make it better.'); giving reassurance and support ('reassured the patient that we are here to support him'); and identifying the pros and cons of the patient's behaviour, for example 'warning of the likely consequences of their actions'. This theme also included the way that participants described communication in the de-escalation context including tone of voice (e.g., 'using a calm tone'), the use of body language during de-escalation, (e.g., 'kept eye contact, open, friendly body language').

Tactics. This theme encompassed the actions that might be used to defuse a situation. The most frequently identified tactic was creating space for the patient, most commonly by moving the him/her away from the situation, for example to a quiet room. Distraction tactics were cited and sometimes unspecified ('Used distraction techniques'), but also encompassed concrete examples of refocusing the patient on an alternative activity ('use of voice to distract', 'offering a drink', 'offering a newspaper', and 'playing chess'). Supporting patients to use their own learned coping strategies, for example 'breathing techniques to calm down', were also described. 
Getting help. This theme comprised the coded items that related to seeking support from other staff, i.e., calling for help, and the use of personal alarms to get the attention of colleagues.

Assessment / risk. This theme included the use of assessment of the situation to identify possible courses of action, the knowledge and information used to make this assessment ('used knowledge of the patient and his family history to talk to the patient with the hope that he would see things more positively').

Interpersonal skills. This theme comprised elements that described the interpersonal skills used during de-escalation, such as showing empathy and the use of humour.

Containment measures. This theme comprised the actions participants described as deescalation interventions that were more coercive in nature than those under other thematic headings in order to gain compliance or reduce risk, and included administration of oral PRN medication, physical interventions, seclusion and enhanced observations.

\section{Responses to vignettes}

Responses concordant with each of the six high level themes were identified in participants' written responses to each of the four aggressive scenarios; all responses could be assigned to at least one theme. Figure 1 shows the frequency of responses under each theme by type of aggression.

Figure 1 about here

Communication. Communication strategies varied according to the type of aggression described. Problem-solving featured prominently in response to VA $(n=54,75 \%)$, e.g., 'Encourage him to go to another area to watch TV', but not in response to the AP vignette $(n=0,0 \%)$. Instructions were reported as appropriate responses to all types of aggression, but most commonly in the case of AP $(n=45,63 \%)$. Enforcing of boundaries was the most cited instruction in response to $\operatorname{veVA}(n=12,17 \%)$ e.g., 'Inform him that his behaviour is not acceptable'. Gathering information from the patient was cited by almost half of participants in 
response to self-directed aggression $(n=31,43 \%)$, whilst nineteen $(26 \%)$ participants wanted to find out more about the causes in the vignette describing AO. Offering reassurance or support was reported in response to all types of aggression. The purposeful use of voice was identified in response to all types of aggression although this formed a small minority of responses across vignettes (range 3-9, 4-13\%); and use of body language was not mentioned in response to any scenario. Talking calmly was cited by participants in response to all types of aggression (range $1-8,1-11 \%)$, though only by one participant in response to AP. One participant (1\%) stated the need to 'talk in an authoritative firm voice' with the verbally aggressive patient. Two participants (3\%) would have used a raised voice to communicate with the patient who was displaying AP, and one (1\%) would have used a 'commanding tone'.

Tactics. The most frequently reported tactic in response to VA and AP was creating a safe space for de-escalation; most commonly by moving the aggressor to a quieter area. Two participants reported that they would stand between the VA patient and his target to create a barrier. Distraction was a cited tactic in response to $\mathrm{SH}(n=10,14 \%)$, usually in the form of offering activities, e.g., 'distract her with something else she likes doing'. In response to the AO vignette, observation and monitoring before further action was identified by nine (13\%), and ignoring the behaviour by three (4\%), e.g., 'ignore the behaviour but observe'; observation and monitoring was also a selected response in the case of $\mathrm{SH}(n=7,10 \%)$. Time out was reported as the appropriate strategy in response to $\mathrm{AO}(n=4,6 \%)$, and advising the use of coping strategies for $\mathrm{SH}(n=3,4 \%)$, e.g. 'explore other coping skills she can employ other than head butting'.

Getting help. Responses in this theme were reported by participants in response to all four vignettes: $\mathrm{VA}(n=3,4 \%), \mathrm{SH}(n=17,24 \%)$, aggrAP $(n=54,76 \%)$ and $\mathrm{AO}(n=9,13 \%)$. The methods of getting support included shouting or using personal attack alarms.

Assessment. Appropriate assessment actions were identified in different ways; in response to VA two participants stated they would assess, one who would assess the situation 
'with the team', and the other who gave the concise response 'Assess. Problem solve.' Two participants also wanted to assess the AP scenario, both following the same course of action by raising the alarm (i.e., getting help), and then assessing the situation before using restraint if necessary. Risk assessment was deemed necessary by two participants in response to SH, one focused on establishing the safety of staff entering the room, the other on determining the safety of the patient. Risk issues during the de-escalation were described, for example one participant reported would that they would 'maintain a safe distance'. in response to AP. Transfer of information was relatively important in terms of frequency of responses to the SH vignette; $n=21(29 \%)$ participants stated that they would pass on information about the incident to other staff either verbally or via patient documentation and gather information from other sources including care plans or other staff. Timing of interventions was reported by some participants as important, and appeared particularly relevant in response to AO where $n=39$ (54\%) participants either identified the need to respond immediately or, conversely, needing to delay intervention either by finishing their phone call first $(n=11,15 \%)$ or waiting until the patient had calmed down $(n=12,17)$. One participant $(1 \%)$ stated that they would deal with the situation immediately and two (3\%) said that they would only intervene if the situation escalated.

Interpersonal skills. A variety of interpersonal skills were identified in response to VA, including showing empathy $(\mathrm{n}=1,1 \%)$, deploying particular staff with a good rapport with the patient $(\mathrm{n}=1,1 \%)$, and having a calm or friendly manner $(\mathrm{n}=2,3 \%)$. Similar interpersonal skills were identified when responding to $\mathrm{SH}$ : showing interest or concern $(\mathrm{n}=2,3 \%)$, being kind or open $(n=2,3 \%)$, and deploying a staff member with a good rapport with the patient $(n=2,3 \%)$. Interpersonal skills were not identified in response to AP, and only once when responding to AO.

Containment measures. In response to the VA vignette one participant suggested offering oral PRN medication, whilst two staff nurses would have acted similarly in response to 
AP and AO. Five participants suggested PRN in response to SH. No physical interventions were identified in response to the scenario about VA, whilst in response to AO two participants (3\%) identified that physical intervention might be warranted if all other interventions were unsuccessful ('Use of PMAV if verbal intervention not effective and only as a last resort'). Four participants (6\%) would have used physical intervention in response to SH such as 'Guide to sit down', and 'use low level restraint', whilst three (4\%) would have used restraint 'only if the situation escalated'. Physical intervention was suggested as a first-line response to AP on 14 (20\%) occasions, whilst two participants (3\%) would 'consider restraint' and two (3\%) would use it 'only if necessary'. Twenty-one people (30\%) would use or consider restraint if telling the aggressor to stop or let go was unsuccessful. One participant would have taken the patient to seclusion in response to aggression towards others; similarly one participant would have segregated the patient in response to aggression towards objects ("put her in isolation if the behaviour persists'). Enhanced observations were only identified in response to $\mathrm{SH}$; three people (4\%) wanted to review her observation levels, whilst two (3\%) stated that they would raise her observation levels from intermittent to constant observations.

\section{De-escalation interventions}

Seventy one (99\%) participants endorsed talking in a calm manner as a de-escalation intervention, and only two (3\%) did not agree that moving the patient constituted de-escalation (see Figure 2). Restraint as a de-escalation intervention was endorsed by five (7\%), all of whom wrote qualifying comments, for example 'in some circumstances'. Administration of oral PRN medication as a de-escalation intervention split the opinion of participants, with both $n=34(47 \%)$ endorsing and rejecting that this was a de-escalation intervention. Participants were invited to add additional de-escalation interventions that were not listed with the following results: distraction or activities, active listening, ask about medication, encourage going to room, encouraging patient coping skills, reduce stimuli, time off the ward and using a jovial manner. 
Figure 2 about here

Finally, participants were asked to list the three most effective and the three most frequently used interventions. The most used interventions were ranked similarly to those deemed the most effective interventions. Calm talk was the most reportedly effective and the most frequently used intervention, while moving the patient and spending one-to-one time were both endorsed as most commonly used and effective by more than half of respondents. No participant identified giving a risk warning or administering IM medication as either an effective or frequently used de-escalation intervention.

\section{Discussion}

The current study suggests general consensus among clinical staff working on low and medium secure wards of a large psychiatric hospital about the nature of de-escalation; further, that their understanding, with some important exceptions, is in accord with the literature on the subject. Most respondents identified the objective of de-escalation, namely to calm down or prevent escalation/crisis; almost half described specific interventions to be used (e.g., verbal and non-verbal communication skills, non-confrontational limit setting), and the characteristics of the person and situation being de-escalated (e.g., escalation phase, violent situation). These are common elements in definitions of de-escalation found in the literature (CRAG Working Group on Mental Illness, 1996; Cowin et al., 2003).Themes emerging from our analysis were largely concordant with those in both theoretical models of de-escalation and in the wider deescalation literature. Communication is one of the key skills taught in staff training in aggression management training programs (Livingston et al., 2010) This was reflected by our participants' responses; communication was cited as being used / witnessed as a de-escalation intervention by respondents more than any other intervention, and was the most frequently cited in response to clinical vignettes. Calm talk was almost universally accepted as a deescalation intervention by respondents in this study. Conversely, poor staff interpersonal skills are perceived to be a cause of aggression by both staff and patients (Sheridan et al., 1990; 
Spokes et al., 2002; Hallett et al., 2014), whilst showing empathy, care and humour, and calmness are all perceived as important facets of de-escalation, both in this study and in the wider literature (National Institute for Clinical Excellence, 2005; Price \& Baker, 2012). Similarly, creating a safe space for de-escalation to take place was a recurrent theme throughout this study, for example most participants agreed that moving or re-locating the patient or other patients in the vicinity could be a de-escalation intervention, and was thus in accord with the wider literature (Leadbetter \& Paterson, 1995; Dix \& Page, 2008).

The issue of when to intervene was identified by a majority of participants in this study in response to the physical aggression against objects vignette. The only other explicit mention of timing was by one participant in response to the vignette based on aggression towards self. Nonetheless, knowing at what point intervention is necessary, if at all, is seen as an important skill (Mackay et al., 2005). It may be that immediate intervention would be the response of the participants in most situations, as reflected by the results of this study. Alternatively, timing could play a larger role in real-life situations, and it may be that the ecological validity of vignettes is less than ideal as regards this issue. Further investigation of the role of timing in de-escalation is warranted to investigate whether this good practice can be taught in training, or whether 'timing' has an ineffable quality that is only learnt through experience. Research could identify the characteristics of those who are skilled at timing their intervention optimally.

The major divergence between de-escalation interventions reported in the current study and those described in the wider literature was in the use of what might more properly be termed as containment measures. Offering oral PRN medication was identified as a used or witnessed de-escalation intervention by a small but significant minority; further, some participants reported that they would offer PRN medication in circumstances described in three of the four vignettes; half the respondents believed that oral PRN medication could be a deescalation intervention. Only registered nursing staff can administer medication, and, of these, almost two thirds described oral PRN as a de-escalation intervention. Oral PRN medication can 
more accurately be considered a secondary prevention measure, one to be offered to patients prior to the crisis stage of the assault cycle in order to prevent further escalation of aggression; on balance it should not routinely be used as a first-line response, particularly to low levels of aggression as described in our vignettes (National Institute for Clinical Excellence, 2005). All medication use carries an inherent risk; benzodiazepines such as lorazepam and diazepam are often the drugs of choice for PRN use in behaviour management, and aside from potential side effects have a high risk of dependency (Duxbury \& Baker, 2004). Given these risks, oral PRN should be offered when less invasive interventions, such as talking, moving the patient and other de-escalation strategies have failed (Usher \& Luck, 2004). Despite the risks, oral PRN appears to play an important role in conflict management, with research suggesting that the most common conflict behaviour is verbal aggression, which is then contained either with deescalation or PRN medication (Bowers et al., 2013). The current study suggests that a significant proportion of clinical staff, including registered nursing staff, believe it to be a useful first line intervention. Further work is required to educate and change practice in this area. While the number of participants advocating use of coercive physical measures was smaller than that advocating PRN it was still of concern that a fraction of clinical staff appear to misunderstand the core ideas at the heart of de-escalation. We propose that trainers and educationalists devise and deliver clear messages around de-escalation interventions that clearly delineate the nature and role of de-escalation from more coercive techniques; while such techniques may, legitimately, constitute management interventions in some circumstances they should be seen as belonging to a different order of activity from de-escalation. The results of the current study suggest that different types of aggression are perceived to require different interventions. In response to verbal aggression and physical aggression towards others, the suggested interventions mirrored the type of aggression such that most participants suggested verbal interventions to manage verbal aggression, whilst over half suggested some form of physical intervention in the management of physical aggression, even 
when the aggression was not person-directed. This suggests that training in management of aggression and violence should aim to teach techniques where clinical staff aim actively and mindfully to 'step out' of a position of mirroring physical aggression with physical intervention and adopt a 'talk first' mentality.

One of the more interesting results of the current study was our finding that the widest range of de-escalation tactics, and the greatest variety of interventions, were reported in response to self-directed aggression. This could be because the participants thought more creatively about how to manage this type of aggression, that there is more variation in the way self-harm is managed by staff, or that they perceive less or no personal threat and so are freer to be creative. Alternatively, there may be less clear guidance on, and training in, how to manage self-harm with the result that the responses vary more greatly. This is reflected in NICE guidelines on the management of self-harm, which describe medical management in the immediate aftermath of self harm, and possible long-term strategies to reduce reoccurrence, including pharmacological interventions and therapies (National Institute for Clinical Excellence, 2004) but do not describe primary, de-escalation interventions that may be effective in self-harm management. There is therefore a need to identify best practice for the de-escalation of individuals who may go on to engage in self-harm, and to test interventions in evaluative studies.

\section{Limitations}

Clinical vignettes are a relatively systematic and efficient method of data collection but they are not without their limitations. Vignettes alone can have poor external validity, and we did not, in this study, undertake any further data collection such as observations of the phenomena to assess the external validity (Lanza, 1988). However, the internal validity of the vignettes in this study is more robust, with information for the scenarios coming from valid and reliable literature on the subject, and being reviewed to ensure uniformity across the scenarios (Gould, 1996). A second criticism of vignettes is that findings may have low ecological 
validity, not being generalisable outside of the specific vignette scenarios (Hughes \& Huby, 2002). This means that generalisation of the findings of the study outside the boundaries of the scenarios described should be approached with caution. Most of the participants in this study came from one hospital trust, therefore the views expressed are likely to be based on the training they have received and so may not be generalisable to clinical staff in other trusts, particularly where training differs greatly. Further use of the survey in other hospital trusts is needed to investigate the generalisability of these results.

\section{Implications and future considerations}

De-escalation is an important tool in the short-term management of violence and aggression, advocated by best practice guidelines as the preferred first line intervention. Despite this, last resort measures were advocated by some participants as the first line intervention in scenarios describing low level physical aggression. Further empirical studies are needed to investigate how staff de-escalate in practice, and to identify what constitutes effective de-escalation. 


\section{References}

Bowers, L. (2013) The Safewards model. Presentation at North Western Mental Health, Melbourne, Australia, October. http://www.youtube.com/watch?v=vKWE0WPISN0 Accessed $11^{\text {th }}$ September 2014.

Bowers, L. (2014a). A model of de-escalation: Len Bowers provides advice, based on the latest research, on the safest way for staff to deal with conflict and aggression. Mental Health Practice 17(9): 36-37.

Bowers, L. (2014b). Safewards: A new model of conflict and containment on psychiatric wards. Journal Of Psychiatric And Mental Health Nursing 21(6): 499-508.

Bowers, L., James, K., Quirk, A., Wright, S., Williams, H. and Stewart, D. (2013). Identification of the "minimal triangle" and other common event-to-event transitions in conflict and containment incidents. Issues in Mental Health Nursing 34(7): 514-523.

Braun, V. and Clarke, V. (2006). Using thematic analysis in psychology. Qualitative Research in Psychology 3(2): 77-101.

Cowin, L., Davies, R., Estall, G., Berlin, T., Fitzgerald, M. and Hoot, S. (2003). De-escalating aggression and violence in the mental health setting. International Journal of Mental Health Nursing 12(1): 64-73.

CRAG Working Group on Mental Illness, N. H. S. i. S. (1996). The prevention and management of aggression : A good practice statement. Edinburgh, Scottish Office, National Health Service in Scotland.

Dix, R. and Page, M. J. (2008). De-escalation. Psychiatric intensive care.2nd. In: Beer, D., Pereira, S. and Paton, C. (Eds). Cambridge, Cambridge University Press: 24-31.

Duxbury, J. A. and Baker, J. (2004). The use and nursing management of benzodiazepines in acute, mental health inpatient care: A discussion. Journal Of Psychiatric And Mental Health Nursing 11(6): 662-667.

Gould, D. (1996). Using vignettes to collect data for nursing research studies: How valid are the findings? Journal of Clinical Nursing 5(4): 207-212.

Hallett, N., Huber, J. and Dickens, G. (2014). Violence prevention in inpatient psychiatric settings: Systematic review of studies about the perceptions of care staff and patients Aggression and Violent Behavior 19(5): 502-514.

Hughes, R. and Huby, M. (2002). The application of vignettes in social and nursing research. Journal of Advanced Nursing 37(4): 382-386.

Lanza, M. L. (1988). Technical notes: Development of a vignettea data collection instrument about patient assault. Western Journal of Nursing Research 10(3): 346-351. 
Leadbetter, D. and Paterson, B. (1995). De-escalating aggressive behaviour. Management of violence and aggression in health care. In: Kidd, B. and Stark, C. (Eds). London, Gaskell/Royal College of Psychiatrists: 49-84.

Livingston, J. D., Verdun-Jones, S., Brink, J., Lussier, P. and Nicholls, T. (2010). A narrative review of the effectiveness of aggression management training programs for psychiatric hospital staff. Journal of Forensic Nursing 6(1): 15-28.

Mackay, I., Paterson, B. and Cassells, C. (2005). Constant or special observations of inpatients presenting a risk of aggression or violence: Nurses' perceptions of the rules of engagement. Journal Of Psychiatric And Mental Health Nursing 12(4): 464-471.

McDonnell, A. A. (2010). Managing aggressive behaviour in care settings: Understanding and applying low arousal approaches. London, Wiley.

National Institute for Clinical Excellence (2004). The short-term physical and psychological management and secondary prevention of self-harm in primary and secondary care. London, NICE.

National Institute for Clinical Excellence (2005). The short-term management of disturbed/violent behaviour in in-patient psychiatric settings and emergency departments. London, National Institute for Clinical Excellence.

Paterson, B., Leadbetter, D. and Miller, G. (2004). Workplace violence in health and social care as an international problem: A public health perspctive on the 'total organisational response'. http://www.nm.stir.ac.uk/documents/ld-integrated-response.pdf. Accessed: 12th November 2012.

Price, O. and Baker, J. (2012). Key components of de-escalation techniques: A thematic synthesis. International Journal of Mental Health Nursing 21(4): 310-319.

Richter, D. (2006). Non-physical conflict management and de-escalation. Violence in mental health settings: Causes, consequences, management. In: Richter, D. and Whittington, R. (Eds). New York, Springer Science and Business Media: 125-144.

Roberton, T., Daffern, M., Thomas, S. and Martin, T. (2012). De-escalation and limit-setting in forensic mental health units. Journal of Forensic Nursing 8(2): 94-101.

Sheridan, M., Henrion, R., Robinson, L. and Baxter, V. (1990). Precipitants of violence in a psychiatric inpatient setting. Hospital and Community Psychiatry 41(7): 776-780.

Spokes, K., Bond, K., Lowe, T., Jones, J., Illingsworth, P., Brimblecombe, N. and Wellman, N. (2002). Hovis--the hertfordshire/oxfordshire violent incident study. Journal of Psychiatric and Mental Health Nursing 9(2): 199-209.

Stevenson, S. (1991). Heading off violence with verbal de-escalation. J Psychosoc Nurs Ment Health Serv 29(9): 6-10.

Usher, K. and Luck, L. (2004). Psychotropic prn: A model for best practice management of acute psychotic behavioural disturbance in inpatient psychiatric settings. International Journal of Mental Health Nursing 13(1): 18-21. 
Yudofsky, S. C., Silver, J. M., Jackson, W., Endicott, J. and Williams, D. (1986). The overt aggression scale for the objective rating of verbal and physical aggression. The American Journal Of Psychiatry 142(1): 35-39. 
Table 1. Participants' demographic details*

\begin{tabular}{ll}
\hline Gender & $35(48.6 \%)$ \\
Male & $36(50.0 \%)$ \\
Female & $8(11.1 \%)$ \\
\hline Age & $21(29.2 \%)$ \\
$18-24$ & $19(26.4 \%)$ \\
$25-34$ & $17(23.6 \%)$ \\
$35-44$ & $7(9.7 \%)$ \\
$45-54$ & $32(44.4 \%)$ \\
55+ & \\
\hline Role & $32(44.4 \%)$ \\
Non-registered staff (Health & \\
$\quad$ Care & \\
assistant (HCA)) & \\
Qualified staff (Staff nurse, \\
$\quad$ senior staff nurse, deputy \\
$\quad$ ward manager, ward & \\
$\quad$ manager) & \\
Other & \\
\hline Experience & \\
0-4 years & \\
5-9 years & $21(11.1 \%)$ \\
10+ years & $21(29.2 \%)$ \\
\hline Location & $29(40.3 \%)$ \\
Locked ward & \\
Low secure & \\
Medium secure & \\
Various & \\
\hline
\end{tabular}

*Not all totals are $100 \%$ due to missing data 


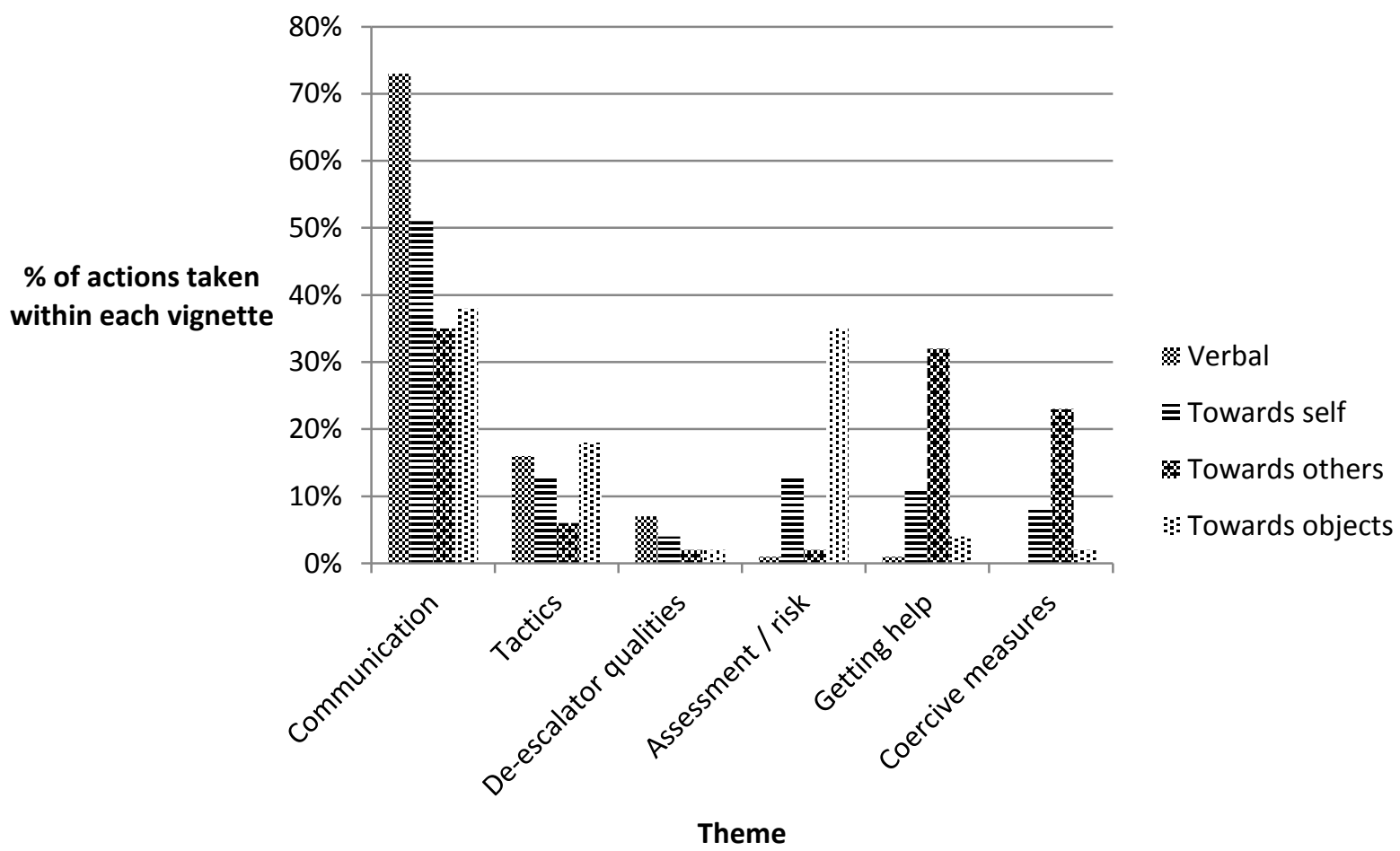

Figure 1. Responses to different types of aggression by theme 


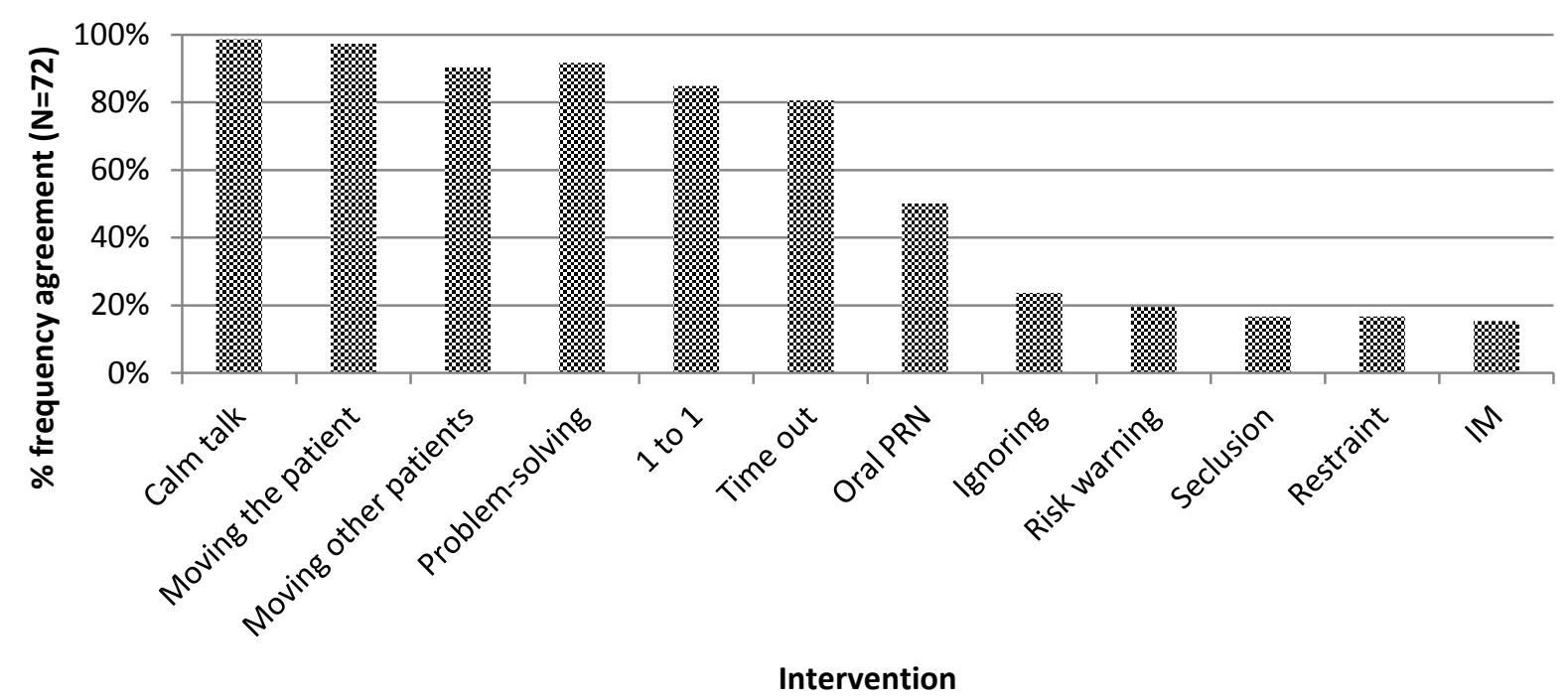

Figure 2. Participants' endorsement of interventions as de-escalation interventions 